\title{
Estimating changes in species abundance from occupancy and aggregation
}

\author{
Cang Hui ${ }^{\mathrm{a}, *}$, Carmen Boonzaaier ${ }^{\mathrm{b}}$, Luz Boyero ${ }^{\mathrm{c}, \mathrm{d}}$ \\ ${ }^{a}$ Centre for Invasion Biology, Department of Botany and Zoology, University of Stellenbosch, Private Bag X1, Matieland 7602, South Africa \\ ${ }^{\mathrm{b}}$ Department of Conservation Ecology and Entomology, University of Stellenbosch, Private Bag X1, Matieland 7602, South Africa \\ ' Wetland Ecology Department, Doñana Biological Station-CSIC, Av. Americo Vespucio s/n, 41092 Sevilla, Spain \\ ${ }^{\mathrm{d}}$ School of Marine and Tropical Biology, James Cook University, Townsville, Qld 4811, Australia
}

\begin{abstract}
Predicting the change in abundance is pivotal for evaluating species' current conservation status and population viability. Empirical works have suggested that species with an increasing abundance have a more aggregated distribution than those with a declining abundance (namely, the change-aggregation hypothesis, $\mathrm{CAH}$ ). Here we introduced an improved negative binomial distribution model of the occupancy-abundance relationship (OAR) to estimate the change in abundance from changes in occupancy or aggregation. Analysis of the model suggests that (i) in general the change in abundance is synchronized with the change in occupancy when the level of environmental heterogeneity remains constant, and (ii) there could exist a threshold of the population density above which the CAH is no longer valid. Tests using data of epigaeic ants in Fynbos of South Africa collected from different seasons and macro-invertebrates from different localities in streams of central Spain verified these model propositions and thus support the use of this model as a monitoring method for assessing species persistence. Results suggest that the change in abundance can be estimated from the change in occupancy often obtained from cost-efficient presence-absence records, and a revision of the traditional $\mathrm{CAH}$ is necessary to capture the threshold phenomenon in the change-aggregation relationship. This work thus signifies the use of the three distinct but related concepts of population structure (i.e. occupancy, abundance and aggregation) in conservation biology.
\end{abstract}

\section{Zusammenfassung}

Vorhersagen zur Abundanzdynamik sind entscheidend für eine Bewertung des aktuellen Gefährdungsgrades von Arten und der Überlebensfähigkeit von Populationen. Empirische Arbeiten legen nahe, dass Arten mit zunehmender Siedlungsdichte ein stärker aggregiertes Verteilungsmuster haben als Arten, deren Abundanz zurückgeht (das ist die "change-aggregation-Hypothese, $\mathrm{CAH})$.

Wir stellen hier ein verbessertes Modell der Besetzungs-Abundanz-Beziehung (occupancy-abundance relationship: OAR) mit negativer Binominalverteilung vor, um Abundanzänderungen auf Basis von Änderungen der Besetzung und der Aggregation abzuschätzen. Die Analyse des Modells legt nahe, dass (1) im Allgemeinen die Abundanzänderung mit einer Änderung der Besetzung synchronisiert ist, wenn der Grad der Umweltheterogenität konstant bleibt, und dass (2) es einen Schwellenwert geben könnte, oberhalb dessen die CAH nicht länger gültig ist.

\footnotetext{
${ }^{*}$ Corresponding author. Tel.: +27 21808 3413; fax: +27 218082995 .

E-mail address: chui@sun.ac.za (C. Hui).
} 
Tests mit Daten zu epigäischen Ameisen aus verschiedenen Jahreszeiten (Fynbos, Südafrika) und zu Makro-Invertebraten von unterschiedlichen Sammelstellen in Flüssen (Zentral-Spanien) bestätigten die Modellannahmen und unterstützen daher den Nutzen unseres Modells als eine Monitoringmethode um die Persistenz von Arten abzuschätzen. Die Ergebnisse legen nahe, dass Abundanzänderungen aus Änderungen der Besetzung abgeleitet werden können, die man oft aus kostengünstigen An/Abwesenheitsdaten erhält. Auch scheint es nötig, die traditionelle CAH zu überarbeiten, um das Schwellenwert-Phänomen in der Wechsel-Aggregations-Beziehung zu erfassen. Die vorliegende Arbeit deutet den Nutzen der drei unterschiedlichen, aber verwandten Konzepte der Populationsstruktur (Besetzung, Abundanz und Aggregation) für die Naturschutzbiologie an. (C) 2012 Gesellschaft für Ökologie. Published by Elsevier GmbH. All rights reserved.

Keywords: Aggregation; Occupancy-abundance relationship; Spatiotemporal dynamics; Change-aggregation hypothesis; Epigaeic ants; Stream invertebrates

\section{Introduction}

Abundance (or population size) is often described by its first-moment of the number of individuals from multiple samples (i.e. the mean population density). Because it is an important indicator of population viability, understanding the spatiotemporal dynamics of abundance becomes critical for assessing the extinction risks of many rare species. Tracing back to the dawn of ecology, mechanistic models have often been used to study the spatiotemporal dynamics of abundance based on the life history of population growth and dispersal (Holmes 1993; Skellam 1951). However, the application of such models to quantify changes in abundance is limited because: (i) life-history parameters, as model input, are more difficult to obtain than directly monitoring population dynamics itself, and (ii) environmental dynamics and heterogeneity are often ignored for deducting mathematically analytic conclusions. Moreover, it remains a challenge to disentangle the source of population dynamics - whether the variation in abundance is caused by life-history adaptation, density dependence, dispersal, environmental change, or a combination of these factors and their interactions. Thus, there is an urgent requirement for models that use standard, cost-efficient data captured from ecological surveys and that enable straightforward inference of changes in abundance.

Recently, the zeroth and second-moments of population size (that is, the occupancy and the variance of abundance) have received much attention as they can provide further information regarding species distribution. Specifically, species' occupancy, derived mostly from the cost-efficient presence-absence maps (Brotons, Thuiller, Araujo, \& Hirzel 2004; Gaston \& Fuller 2009), depicts the proportion of samples with more than one individual and has been shown to hold a positive correlation with species abundance, known as the occupancy-abundance relationship (OAR) (Gaston \& Blackburn 2000; Holt, Gaston, \& He 2002). This positively correlated OAR implies that widespread species (often niche generalists) are more abundant than narrowlydistributed species (often niche specialists) (Brown 1984; Gaston, Blackburn, \& Lawton 1997). The variance of abundance (i.e. the second-moment), on the other hand, depicts the degree of over-dispersion in samples and is important for quantifying patterns of aggregated species distribution (Fortin \& Dale 2005; Hui, Veldtman, \& McGeoch 2010). The variance of abundance has often been reported as a power function of the mean population density, namely Taylor's power law (TPL) (Kendal 2004; Perry 1981; Taylor 1961). The progress in clarifying the interrelationship between occupancy, mean and variance of abundance could potentially provide an alternative way of forecasting the changes of population size.

One important application of the OAR is to estimate species abundance from occupancy (Gaston, Borges, He, \& Gaspar 2006; He \& Gaston 2000; Hui et al. 2009). This is appealing because measuring occupancy requires much less sampling effort than counting abundance. However, a more relevant issue for conservation management would be whether the change in abundance could be estimated from the change in occupancy. This is a different question because abundance and the change in abundance are often determined by different demographic and environmental factors (Roura-Pascual, Bas, \& Hui 2010). For instance, high abundance could indicate a large carrying capacity of the environment, whilst rapid change in abundance may imply habitat degradation or loss. A well-known hypothesis, namely the change-aggregation hypothesis (CAH hereafter) states that species in decline have sparse distributions whereas expanding species tend to have highly aggregated distributions (Johnson 1998; Rodriguez \& Delibes 2002; Shigesada, Kawasaki, \& Teramoto 1979). This is because declining species retract their range to spatially isolated habitat 'islands' (Johnson 1998), whereas species often expand through distance-limited colonization (Hanski \& Gyllenberg 1997). Although lacking theoretical confirmation, the CAH has proved fruitful especially for monitoring species trends (Wilson, Thomas, Fox, Roy, \& Kunin 2004). Using the OAR and TPL as bases for extrapolating changes in species abundance will not only provide a theoretical underpinning for this $\mathrm{CAH}$, but will also be of practical importance for cost-efficient monitoring in biodiversity conservation.

Here, we explore the potential of using OAR models for depicting species spatiotemporal dynamics and provide a mechanistic understanding of the relationship between the change in abundance and the change in occupancy. This 
enables us to further revise and refine the CAH. Specifically, using two datasets (an epigaeic ant community in Fynbos from different seasons (South Africa) (Boonzaaier, McGeoch, \& Parr 2007) and a macro-invertebrate community in streams from different localities (Spain) (Boyero 2003)), we test the robustness and strength of the relationship between changes in abundance and occupancy. The CAH is revised, and a general form of the change-aggregation relationship proposed. We advocate the use of this relationship as a practical technique to estimate changes in species abundance when assessing the conservation status and population viability.

\section{Materials and methods}

\section{The relationship between changes in abundance and occupancy}

Species distributions are rarely random but rather overdispersed (or aggregated) due to either habitat heterogeneity or local intra- or inter-specific interactions (Fahrig \& Nuttle 2005). When species are over-dispersed, the number of individuals in samples can be described by a negative binomial distribution (NBD; He and Gaston, 2000; Pielou 1977; Wright 1991). This yields an OAR model for aggregated species distributions, $p_{+}=1-(1+\mu / k)^{-k}$, where $p_{+}$ is the occupancy, $\mu$ the mean abundance and $k$ a clumping parameter of the species' distribution indicating from highly over-dispersed at $k=0$ to random at $k=+\infty$ which converges to a Poisson model $\left(p_{+}=1-e^{-\mu}\right)$ (Wright 1991; for convienience, a description of all symbols used is listed in Table 1). Although this NBD model provides a better fit to the observations than the Poisson model, it entails a quadratic relationship between the mean and variance of abundance, $\sigma^{2}=\mu+\mu^{2} / k$, in contrast to the well-observed Taylor's power-law (TPL) form $\sigma^{2}=c \mu^{b}$ ( $c$ and $b$ are constants) (Perry 1981; Taylor 1961). To correct this inconsistency, $\mathrm{He}$ and Gaston (2003) substitute the clumping parameter with a transformation of the variance $\left(k=\mu^{2} /\left(\sigma^{2}-\mu\right)\right)$ and derive the following improved NBD model for the OAR (Gaston et al. 2006), $p_{+}=1-\left(c \mu^{b-1}\right)^{\mu /\left(1-c \mu^{b-1}\right)}$. This improved NBD model implies that the number of individuals follows a constrained NBD, with its mean-variance relationship complying with Taylor's power law. Although this use of probability distributions as a platform for describing the OAR represents a significant advance, the NBD model still lacks ability to forecast demographic and distributional changes.

To examine the changes in abundance and occupancy, we allow the occupancy and mean abundance to be a function of an independent variable, $T$; specifically it represents time and space (localities) in the following way. If the sampling time or locality is changed (denoted as $\partial \tau$ ), the occupancy and abundance will also change accordingly (denoted as
Table 1. Model parameters and their description.

\begin{tabular}{|c|c|}
\hline Symbol & Description \\
\hline$p_{+}$ & $\begin{array}{l}\text { Occupancy, with } \partial p_{+} \text {indicating the change in } \\
\text { occupancy }\end{array}$ \\
\hline$\mu$ & $\begin{array}{l}\text { Mean abundance, with } \partial \mu \text { indicating the change in } \\
\text { mean abundance }\end{array}$ \\
\hline$k$ & $\begin{array}{l}\text { A clumping parameter, ranging from } 0 \\
\text { (over-dispersion) to infinity (random) }\end{array}$ \\
\hline$\sigma^{2}$ & The variance of abundance \\
\hline$c$ & $\begin{array}{l}\text { The variance of abundance when } \mu=1 \text { in the } \\
\text { Taylor's power law }\end{array}$ \\
\hline$b$ & The exponent of the Taylor’s power law \\
\hline$T$ & $\begin{array}{l}\text { An independent variable (time or space) driving the } \\
\text { change in population sizes, with } \partial \tau \text { indicating the } \\
\text { change in this independent variable. The derivative of } \\
p_{+} \text {or } \mu \text { with respect to } \tau \text { (i.e. } \partial p_{+} / \partial \tau \text { or } \partial \mu / \partial r \text { ) } \\
\text { indicates the change in occupancy or abundance due } \\
\text { to the change of } T \text {. That is, different observations of } \\
\text { occupancy and abundance in one locality at different } \\
\text { times ( } \partial r \text { represents the elapse of time), or at the } \\
\text { same time but different localities ( } \partial \tau \text { represents the } \\
\text { change of space). }\end{array}$ \\
\hline$I_{L}$ & Lloyd’s index of patchiness \\
\hline$r$ & Intrinsic population growth rate \\
\hline$K$ & Carrying capacity \\
\hline
\end{tabular}

$\partial p_{+}$and $\partial \mu$, respectively). Therefore, we have the following derivative of species abundance from the improved NBD model,

$$
\begin{aligned}
\frac{\partial \mu}{\partial \tau}= & -\frac{\mu \omega 1 \partial p_{+}}{1-p_{+} \theta \partial \tau}-\frac{\mu^{2}+c \mu^{b} \ln \left(1-p_{+}\right)}{\theta} \\
& \times \frac{1 \partial c}{c \partial \tau}+\ln \mu \frac{\partial b^{-}}{\partial \tau},
\end{aligned}
$$

where $\omega=1-c \mu^{b-1}$ and $\theta=(b-1) \mu+((b-1)-(b-2) \omega)$ $\ln \left(1-p_{+}\right)$. In this model the two TPL coefficients $c$ and $b$ are considered also sensitive to the sampling time and locality $(\partial c / \partial r$ and $\partial b / \partial r)$; that is, species potentially inhabit a heterogeneous or a dynamic environment reflected by the change of their distribution structures $(c$ and $b$ ). However, it is impossible to estimate the change of these two coefficients $(\partial c$ and $\partial b)$ without knowing the change in abundance beforehand. Therefore, to overcome this problem we here consider two simplified scenarios of this model.

First, if we assume a homogenous environment or a heterogeneous one but with the level of heterogeneity remaining constant temporally and spatially (i.e. $c$ and $b$ are constant in Eq. (1)), we have the following improved NBD model:

$$
\frac{\partial \mu}{\partial T}=-\frac{\mu \omega}{1-p_{+} \theta \partial p_{+}}
$$


Second, if the environment is homogenous and the species distribution completely random (i.e. when $c$ and $b$ equal one), we have the following Poisson model:

$\frac{\partial \mu}{\partial \tau}=\frac{1}{1-p_{+} \partial p_{+}}$

Because $p_{+}<1$, it is obvious that the changes in abundance and occupancy for randomly distributed species are synchronized, $\partial \mu / \partial \tau \sim+\partial p_{+} / \partial \tau$. However, to assess the change in abundance $(\partial \mu / \partial \tau)$ for species in homogenous or static heterogeneous environment as in the first scenario (Eq. (2)), knowing only the occupancy $\left(p_{+}\right)$and the change in occupancy $\left(\partial p_{+} / \partial \tau\right)$ is not enough. The abundance of current samples $(\mu)$ is needed.

Downing (1981) observed that the vast majority of estimates for $b$ range between 1 and 2 . This bounded range of the TPL exponent $(b)$ potentially implies that species distributions in space could be self similar and thus can only have a bounded fractal dimension from 1 in a linear shape (e.g. distributions along rivers or coast lines) to 2 that covers the entire landscape, even though the exact relationship between $b$ and the fractal dimension is unclear. Only $2 \%$ of the observations are above 2 , and about $0.6 \%$ below one (Downing 1981). This implies that, for most over-dispersed species $\left(\sigma^{2}>\mu\right.$ and thus $\omega<0$ ), we have $\theta>0$ and $\partial \mu / \partial \tau \sim+\partial p_{+} / \partial \tau$ in Eq. (2), i.e. changes in abundance and occupancy are synchronized as in the Poisson model (Eq. (3)). Only few exceptions $(1>b>0$ or $b>2$ ) show inconsistency in the demographic and distributional changes $\left(\partial \mu / \partial \tau \sim-\partial p_{+} / \partial \tau\right)$. Furthermore, because $\mu^{2}+c \mu^{b} \ln \left(1-p_{+}\right)>0$, the last term in the bracket of Eq. (1), which indicates the change in environmental heterogeneity, determines the synchronicity between species abundance and occupancy. Therefore, we can conclude that, regardless of whether a species distribution is random or aggregated, the change in abundance is positively correlated (i.e. synchronized) with the change in occupancy, as long as the level of the environmental heterogeneity remains constant. However, if the environment is spatially varying or temporally dynamic, the synchronicity will be affected by the change in the environmental heterogeneity.

\section{Change-aggregation hypothesis (CAH)}

A question naturally follows: Do species with an increasing occupancy (or an increasing population size) have a more aggregated distribution than species with a declining occupancy (or abundance)? Here to measure species aggregation, we first used Lloyd's (1967) index of patchiness $I_{L}$ $\left(=1+\left(\sigma^{2}-\mu\right) / \mu^{2}\right)$, which has been widely used in the ecological literature (e.g. Bez 2000). This index measures the probability that two randomly selected individuals will be from the same sample, with $I_{L}=1$ indicating random distributions and $I_{L}>1$ over-dispersion (aggregation), and has been shown as a reliable measure for detecting aggregated structure of species distributions (Hui, Veldtman et al. 2010).
The CAH can be interpreted in two ways: (i) a highly aggregated distribution entails an increase of abundance, $\partial \mu / \partial \tau \sim+I_{L}$; (ii) an increasing level of aggregation entails an increase of abundance, $\partial \mu / \partial \tau \sim+\partial \mathrm{I}_{L} / \partial \tau$. If we use the logistic equation to depict population dynamics $(\partial \mu / \partial r=r \mu(1-\mu) / K)$, where $r$ and $K$ denote the intrinsic growth rate and carrying capacity, respectively), it is straightforward to show that $\partial \mu / \partial \tau \sim+I_{L}$ if $\mu<K / 2$, and $\partial \mu / \partial \tau \sim-I_{L}$ if $\mu>K / 2$ (Pielou 1977). This suggests that the first interpretation of the CAH only applies when the population size is much lower than the carrying capacity (Borregaard \& Rahbek 2006; Wilson et al. 2004; Hui 2011). Here, we focused on examining the second interpretation of the $\mathrm{CAH}$ based on the same framework as above. Following the same procedure, we have the following relationship between the change in abundance and the change in aggregation,

$\frac{\partial \mu}{\partial \tau}=\frac{\mu^{2}}{1-(2-b) c \mu^{b-1} \partial \tau}$

If the mean abundance is lower than a threshold $\mu<(c(2-b))^{1 /(1-b)}$, we have a synchronized abundance and aggregation $\partial \mu / \partial \tau \sim+\partial I_{L} / \partial \tau$ and the CAH is supported. However, if $\mu>(c(2-b))^{1 /(1-b)}$, the synchronicity breaks $\left(\partial \mu / \partial \tau \sim-\partial I_{L} / \partial \tau\right)$ and the $\mathrm{CAH}$ is refuted. Since the change in abundance and the change in occupancy are synchronized (see above), the relationship between the change in occupancy and the change in aggregation can be expected to follow the same threshold phenomenon. Accordingly, we propose a revised CAH where there exists a threshold in the mean abundance of samples $\left(\mu^{*}=(c(2-b))^{1 /(1-b)}\right)$ so that a synchronized change of abundance (or occupancy) and aggregation is expected when the species is rare $\left(\mu<\mu^{*}\right)$, as in the traditional $\mathrm{CAH}$, but the changes lose the synchronicity for common species $\left(\mu>\mu^{*}\right)$.

To further investigate whether or not this threshold $\left(\mu^{*}\right)$ in the revised CAH is index-dependent, we further apply the same procedure as above to another widely-used index of aggregation, namely the coefficient of variation $(\mathrm{CV}=\sigma / \mu)$, a normalized measure of dispersion, and derive the following relationship:

$\frac{\partial \mu}{\partial T}=\frac{2 \mu^{2-b / 2} \partial \mathrm{CV}}{(b-2) c^{1 / 2} \partial T}$

Because we normally have $1<b<2$ (Downing 1981), it is evident that $\partial \mu / \partial \tau \sim-\partial C V / \partial r$, suggesting that (i) there is no threshold when measuring the aggregation using $\mathrm{CV}$ and (ii) changes in abundance and changes in CV are negatively correlated (i.e. an increase of over-dispersion infers a decline of abundance). Therefore, the threshold phenomenon in the revised $\mathrm{CAH}$ can only be detected by specific measures of aggregation (here, Lloyd's $I_{L}$ ) which depict a unique facet of species distributional structure. 

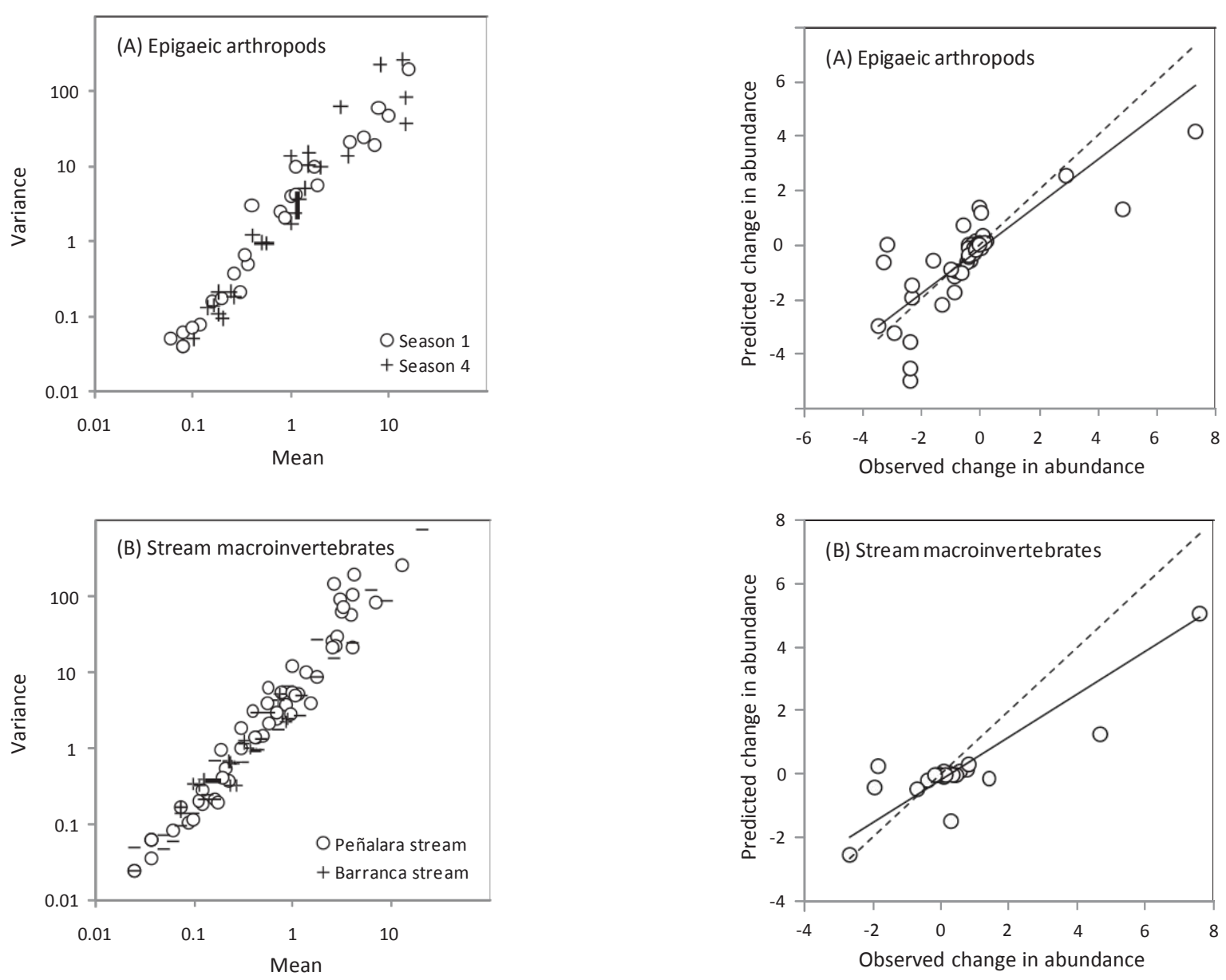

Fig. 1. Power-law relationships between sample mean and variance of abundance for the ants in Fynbos (A) and for the macroinvertebrates in streams of central Spain (B). Points indicate different species in the study.

\section{Empirical evaluation}

We tested the relationship between changes in abundance and occupancy, as well as the revised CAH using two datasets (see Appendix A): one to test population changes through time (Boonzaaier et al. 2007); the other across space (Boyero 2003). The first dataset was taken from a study of the epigaeic arthropods (ant community) in the lowlands of the Cape Floristic Region (Fynbos), where data were collected in 2004 from the Elandsberg Private Nature Reserve $\left(19.03^{\circ} \mathrm{E}\right.$, $33.27^{\circ} \mathrm{S}$ ), Western Cape, South Africa for four seasons. The sample consisted of $10(20 \mathrm{~m} \times 50 \mathrm{~m})$ grids, each containing 10 pitfall traps spaced $10 \mathrm{~m}$ apart. Fifty-nine species and 26,061 individuals were recorded in the four seasons (Boonzaaier et al. 2007). After excluding species that only appeared in one season, 26 species were chosen. Models were tested by using the observed values from the first season (20-25 February) and the last season (8-13 December). The second dataset was taken from the macroinvertebrate community inhabiting two streams, Peñalara stream $\left(40^{\circ} 501\right.$ îN, $\left.3^{\circ} 57 \mathrm{iW}\right)$ and Barranca stream $\left(40^{\circ} 45 \mathrm{i} \mathrm{N}, 4^{\circ} 00 \mathrm{iW}\right)$, in the

Fig. 2. Observed versus predicted change in abundance by the improved negative binomial distribution model (Eq. (2)) for the ants (A) and stream macroinvertebrates (B). Points indicate different species in the study. Solid lines indicate the linear regression fit from the reduced major axis between predictions and observations, whilst dashed lines indicate $y=x$ (i.e. predictions equal observations).

Sierra de Guadarrama, Madrid, Central Spain, with nearly 20,000 individuals from 82 taxonomic groups identified (Boyero 2003). After removing the taxonomic groups that only appeared in one stream or were found only in one sample, 54 taxonomic groups from both streams were selected to test the model.

For testing whether the TPL held for samples from different seasons (the Arthropods) and from different localities (the macro-invertebrates), natural log-transformed data on the mean and variance of abundance were tested using an analysis of covariance (ANCOVA) with seasons and localities being the categorical factor (STATISTICA 10, StatSoft, Inc.).

For testing the relationship between changes in abundance and occupancy, Eqs. (2) and (3) were examined in their ability to estimate the change in abundance from the change in occupancy. Changes were calculated as the differences of abundance and occupancy sampled from different seasons for the ant community and from different streams for the 
Table 2. A summary of statistical results for (i) the improved negative binomial distribution (NBD) model (Eq. (2)) and the Poisson model (Eq. (3)) for estimating change in abundance from change in occupancy and (ii) the change-aggregation hypothesis (CAH) for Lloyd's $I_{L}$ (Eq. (4)) and the coefficient of variation (CV) (Eq. (5)). For the first test (i), intercept and slope (with their 95\% confidence intervals) between observed vs. predicted changes in abundance ( $\partial \mu$ (obs) vs. $\partial \mu$ (pred)) are derived from the Jackknife and bootstrapping using the reduced major axis regression. For testing the CAH, Pearson's $r$ and $p$-value between change in aggregation $\left(\partial I_{L}\right.$ and $\left.\partial \mathrm{CV}\right)$ and change in abundance and occupancy are provided for population density below and above the threshold $\left(\mu^{*}=0.558\right.$ for epigaeic arthropods and $\mu^{*}=0.138$ for stream macroinvertebrates).

\begin{tabular}{|c|c|c|c|c|c|c|c|c|c|}
\hline \multicolumn{2}{|l|}{$\partial \mu$ (obs) vs. $\partial \mu$ (pred) } & Intercept & \multicolumn{2}{|c|}{$95 \%$ CI } & Slope & \multicolumn{2}{|c|}{$95 \%$ CI } & \multirow{2}{*}{$\begin{array}{l}R^{2} \\
0.910\end{array}$} & \multirow{2}{*}{$\begin{array}{l}\text { AIC } \\
13.580\end{array}$} \\
\hline Epigaeic arthropods & Improved NBD & -0.183 & -0.428 & 0.047 & 0.680 & 0.249 & 0.770 & & \\
\hline & Poisson & -0.016 & -0.056 & 0.027 & 0.097 & 0.046 & 0.146 & 0.843 & 34.551 \\
\hline \multirow[t]{2}{*}{ Macroinvertebrates } & Improved NBD & -0.186 & -0.398 & 0.230 & 0.822 & 0.647 & 1.342 & 0.601 & 16.094 \\
\hline & Poisson & -0.036 & -0.076 & 0.044 & 0.132 & 0.097 & 0.231 & 0.446 & 52.620 \\
\hline \multirow[t]{2}{*}{$\mathrm{CAH}$} & & \multicolumn{2}{|l|}{$\partial I_{L}$ vs. $\partial \mu$} & \multicolumn{2}{|l|}{$\partial I_{L}$ vs. $\partial p_{+}$} & \multicolumn{2}{|c|}{$\partial \mathrm{CV}$ vs. $\partial \mu$} & \multicolumn{2}{|c|}{$\partial \mathrm{CV}$ vs. $\partial p_{+}$} \\
\hline & & $r$ & $p$ & $r$ & $p$ & $r$ & $p$ & $r$ & $p$ \\
\hline \multirow[t]{2}{*}{ Epigaeic arthropods } & $\mu \leq \mu^{*}(n=13)$ & 0.053 & 0.864 & 0.163 & 0.595 & -0.132 & 0.668 & -0.501 & 0.081 \\
\hline & $\mu>\mu^{*}(n=13)$ & -0.133 & 0.665 & -0.387 & 0.191 & -0.304 & 0.312 & -0.568 & 0.043 \\
\hline \multirow[t]{2}{*}{ Macroinvertebrates } & $\mu \leq \mu^{*}(n=16)$ & 0.424 & 0.102 & 0.294 & 0.269 & -0.267 & 0.318 & -0.645 & 0.007 \\
\hline & $\mu>\mu^{*}(n=38)$ & -0.050 & 0.768 & -0.468 & 0.003 & -0.153 & 0.358 & -0.668 & $<0.001$ \\
\hline
\end{tabular}

macroinvertebrates. Predictions of mean abundance from the Poisson (Eq. (3)) and the improved NBD model (Eq. (2)) versus observations of the change in mean abundance were regressed using the Jackknife method of the reduced major axis (RMA for JAVA v.1.19; Bohonak \& van der Linde 2004) for mitigating bias in the linear regression (Hui, Terblanche, Chown, \& McGeoch 2010), with the 95\% confidence intervals generated from 10,000 bootstraps. The advantage of using the improved NBD (Eq. (2)) rather than using the Poisson model (Eq. (3)) was also verified by using the Akaike information criterion (AIC $=2 m+n[\ln (\mathrm{RSS} / n)]$, where $m$ is the number of parameters in the model, $n$ the number of observations and RSS the residual sum of squares) (Burnham \& Anderson 2002).

For testing the revised $\mathrm{CAH}$, we examined the relationship between changes in abundance and aggregation, as well as the relationship between changes in occupancy and aggregation. The Pearson correlation was calculated separately for observations of changes in abundance (and occupancy) with their mean abundance below and above the CAH threshold versus aggregation change measured by both Lloyd's $I_{L}$ and the coefficient of variation (CV). The correct sign of the Pearson correlation and its significance from zero were used as evidence to support the revised CAH (Eqs. (4) and (5)).

\section{Results}

The power-law relationship between the mean and variance of abundance was extremely significant for both datasets $\left(R^{2}>0.95, p<0.01\right)$, with the exponent close to $b=3 / 2$ (Fig. 1A and B). The ANCOVA revealed non-significant effects of seasons (Fig. 1A: $F$-ratio $=0.048, p=0.828$ ) and localities (Fig. 1B: $F$-ratio $=3.581, p=0.061$ ), suggesting that it is appropriate to assume a constant level of environmental heterogeneity and thus the use of the improved NBD model (Eq. (2)).

The predicted change in abundance using the improved NBD model (Eq. (2)) was significantly correlated with the observed change in abundance for both datasets $\left(F_{1,24}=72.6\right.$, $p<0.01$, Fig. 2A; $F_{1,52}=80.8, p<0.01$, Fig. 2B). Although the Poisson model (Eq. (3)) also performed significantly better than expected by chance $\left(F_{1,24}=55.1, p<0.01\right.$ for the arthropods in Fynbos; $F_{1,52}=44.3, p<0.01$ for the macroinvertebrates in streams), the AIC scores of the improved NBD model (AIC $=13.6$ and 16.1 for Fig. $2 \mathrm{~A}$ and $\mathrm{B} ; m=4$ ) were significantly lower (chi-square, $p<0.01$ ) than the Poisson model (AIC $=34.6$ and 52.6, respectively; $m=2$ ) (Table 2). The improved NBD model not only performed better than the Poisson model in terms of variance explained, but also presented slopes much closer to one (i.e. predictions equal observations; Fig. 2) than the Poisson model, even though still significantly lower than one for the arthropods; $t$-test, one-tail, $p<0.01$ ) (Table 2).

The revised CAH captured the overall feature of the correlation between changes in abundance and aggregation (Eq. (4)) (the correlation is positive when the mean abundance is below the threshold and negative when above (the aggregation was measured by Lloyd's $I_{L}: \partial I_{L}$ vs. $\partial \mu$ in Table 2; Fig. 3A and C)), and so did it capture the correlation between changes in occupancy and aggregation $\left(\partial I_{L}\right.$ vs. $\partial p_{+}$in Table 2; Fig. $3 \mathrm{~B}$ and $\mathrm{D}$ ). However, the only significant correlation was between the changes in occupancy and aggregation for the macroinvertebrates in streams ( $p<0.01$; Table 2 and Fig. 3D). When the aggregation was measured by the coefficient of variation (CV), all correlations were negative as predicted from Eq. (5) ( $\partial \mathrm{CV}$ vs. $\partial \mu$ and $\partial \mathrm{CV}$ vs. $\partial p_{+}$in Table 2). 

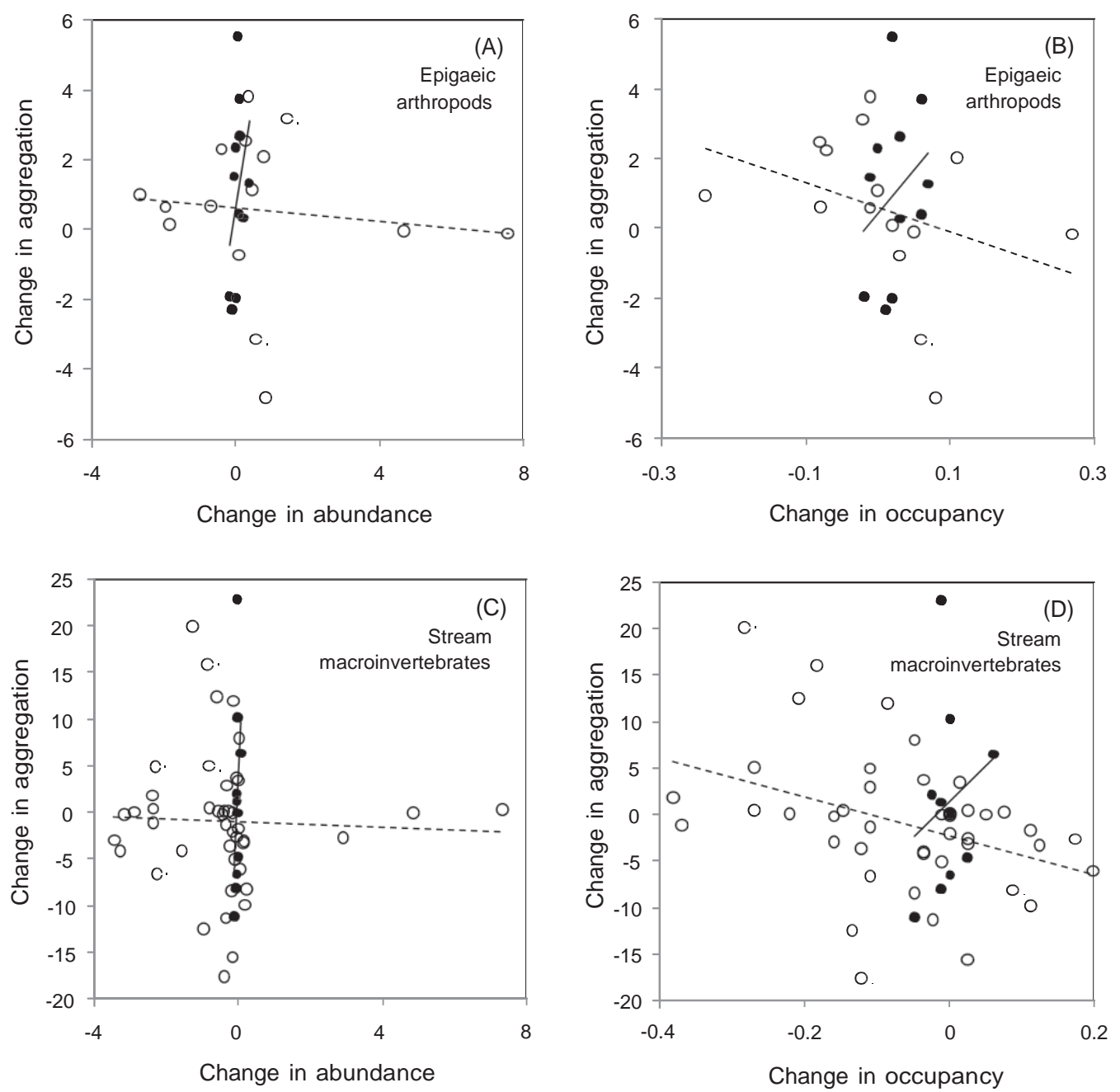

Fig. 3. Relationships between changes in abundance and aggregation (A and B), and between changes in occupancy and aggregation (C and D), where aggregation is measured by Lloyd's $I_{L}$. Dark points and their linear regression lines (solid lines) represent species with their abundance (or occupancy) below the threshold for the revised change-aggregation hypothesis; open circles and their linear regression lines (dashed lines) represent species above the threshold. Both axes are drawn in linear scale.

\section{Discussion}

The improved NBD model presented here showed reliable results for data from different taxonomic groups and geographical regions (Boonzaaier et al. 2007; Boyero 2003). These results support the use of the improved NBD model for extrapolating abundance from occupancy at fine scales (Gaston et al. 2006; He and Gaston, 2003). However, although the improved NBD model showed advantages over the Poisson model in capturing the relationship between changes in occupancy and abundance, both models substantially underestimated the change in abundance (Table 2 and Fig. 2). This might be due to the failure to incorporate changes in environmental heterogeneity for both models (Hui et al. 2009). For instance, it is the stochastic birth-death-dispersal processes that cause the change in non-random structure of species distribution in the improved NBD model (Pielou
1977). In reality, variations in species abundance are driven by a combination of ecological processes and environmental heterogeneity (Fahrig and Nuttle, 2005). Neglecting the effect of environmental heterogeneity thus leads to an underestimation of the demographic change when using the improved NBD model (Hui et al. 2009). Because a stream environment is, in general, more homogenous than a terrestrial environment due to its high connectivity by the flow of water (Wiens 2002), the change in abundance was better estimated for the stream macro-invertebrates than for the ants (Fig. 2C and D). This further supports the idea that environmental heterogeneity could amplify the uncertainty in the model prediction. Future model development for accurately estimating changes in abundance, therefore, lies in incorporating environmental heterogeneity in the OAR model.

The choice of Lloyd's aggregation index of patchiness could, to a certain degree, affect the strength of the revised 
CAH. Lloyd's index of patchiness measures the spatial variance, a statistical measure of over-dispersion among sampling points in a given area (Wiens 2000). Although measures of spatial autocorrelation and other spatially explicit indices could perform differently (Dale et al. 2002; Fortin and Dale, 2005), each index only describes a unique facet of species non-random distributional structure and they all agree with each other at fine scales (Hui, Veldtman et al. 2010). However, choosing another aggregation index (e.g. the coefficient of variation in Eq. (5)) could potentially affect the revised change-aggregation relationship (see Appendix A for relationships between Lloyd's index, the Morisita index and the coefficient of variation). This suggests that only certain aggregation measures are highly sensitive to changes in abundance and occupancy, and highlights the importance of choosing sensitive measures to detect changes in population structure for conservation purposes.

The existence of the CAH threshold could reflect a percolation process of species distribution (Hui \& McGeoch 2007) where the aggregated structure strengthens with the increase of population size but slowly declines once the population size passes beyond the percolation threshold (He and Hubbell 2003; Plotkin, Chave, \& Ashton 2002). The existence of this threshold also implies a two-phase increase of occupancy, as in the range expansion of some invasive species: from an initially low spreading rate to a high rate after a certain lag phase (Crooks 2005). This jump in spreading rate could happen when the population size reaches the percolation threshold, consistent with the revised CAH. Because the two empirical datasets are not ideal for analyzing range expansion, the potential of using the revised $\mathrm{CAH}$ for explaining the two-phase range expansion in invasive species warrants further investigation. Even though long-term data are clearly more appropriate for testing the performance of the improved NBD model and the revised CAH, the current empirical evaluations support the use of these models as an alternative, cost-efficient technique for assessing species change in conservation status from monitoring their aggregation structure.

\section{Acknowledgments}

We are grateful to the comments and logistic help from M.A. McGeoch, S. Harrison, B. Laniewski, C.L. Parr, D.M. Mager, K. Gross and B. Beckage. This work was supported by the NRF Blue Sky Programme and the DST-NRF Centre of Excellence for Invasion Biology.

\section{Appendix A. Supplementary data}

Supplementary data associated with this article can be found, in the online version, at doi:10.1016/j.baae.2012.02.003.

\section{References}

Bez, N. (2000). On the use of Lloyd's index of patchiness. Fisheries Oceanography, 9, 372-376.

Bohonak, A. J., \& van der Linde, K. (2004). RMA: Software for reduced major axis regression, Java version. http://www.kimvdlinde.com/professional/rma.html.

Boonzaaier, C., McGeoch, M. A., \& Parr, C. L. (2007). Fine-scale temporal and spatial dynamics of epigaeic ants in Fynbos: Sampling implications. African Entomology, 15, 1-11.

Borregaard, M. K., \& Rahbek, C. (2006). Prevalence of intraspecific relationships between range size and abundance in Danish birds. Diversity and Distributions, 12, 417-422.

Boyero, L. (2003). Multiscale patterns of spatial variation in stream macroinvertebrate communities. Ecological Research, 18, 365-379.

Brotons, L., Thuiller, W., Araujo, M. B., \& Hirzel, A. H. (2004). Presence-absence versus presence-only modelling methods for predicting bird habitat suitability. Ecography, 27, 437-448.

Brown, J. H. (1984). On the relationship between abundance and distribution of species. American Naturalist, 124, 255-279.

Burnham, K. P., \& Anderson, D. R. (2002). Model selection and multimodel inference: A practical information-theoretical approach. Berlin: Springer-Verlag.

Crooks, J. A. (2005). Lag times and exotic species: The ecology and management of biological invasions in slow-motion. Ecoscience, 12, 316-329.

Dale, M. R. T., Dixon, P., Fortin, M. J., Legendre, P., Myers, D. E., \& Rosenberg, M. S. (2002). Conceptual and mathematical relationships among methods for spatial analysis. Ecography, 25, 558-577.

Downing, J. A. (1981). Spatial heterogeneity: Evolved behaviour or mathematical artifact? Nature, 323, 255-257.

Fahrig, L., \& Nuttle, W. K. (2005). Population ecology in spatially heterogeneous environments. In G. M. Lovett, C. G. Jones, M. G. Turner, \& K. C. Weathers (Eds.), Ecosystem function in heterogeneous landscapes (pp. 95-118). Berlin: Springer.

Fortin, M. J., \& Dale, M. R. T. (2005). Spatial analysis: A guide for ecologists. Cambridge: Cambridge University Press.

Gaston, K. J., \& Blackburn, T. M. (2000). Pattern and process in macroecology. Oxford: Blackwell Science.

Gaston, K. J., Blackburn, T. M., \& Lawton, J. H. (1997). Interspecific abundance range size relationships: An appraisal of mechanisms. Journal of Animal Ecology, 66, 579-601.

Gaston, K. J., Borges, P. A. V., He, F. L., \& Gaspar, C. (2006). Abundance, spatial variance and occupancy: Arthropod species distribution in the Azores. Journal of Animal Ecology, 75, 646-656.

Gaston, K. J., \& Fuller, R. A. (2009). The sizes of species’ geographic ranges. Journal of Applied Ecology, 46, 1-9.

Hanski, I., \& Gyllenberg, M. (1997). Uniting two general patterns in the distribution of species. Science, 275, 397-400.

He, F. L., \& Gaston, K. J. (2000). Estimating species abundance from occurrence. American Naturalist, 156, 553-559.

He, F. L., \& Gaston, K. J. (2003). Occupancy, spatial variance, and the abundance of species. American Naturalist, 162, 366-375.

He, F. L., \& Hubbell, S. P. (2003). Percolation theory for the distribution and abundance of species. Physical Review Letters, 91, 198103. 
Holmes, E. E. (1993). Are diffusion models too simple-A comparison with telegraph models of invasion. American Naturalist, 142, 779-795.

Holt, A. R., Gaston, K. J., \& He, F. L. (2002). Occupancyabundance relationships and spatial distribution: A review. Basic and Applied Ecology, 3, 1-13.

Hui, C. (2011). Forecasting population trend from the scaling pattern of occupancy. Ecological Modelling, 222, 442-446.

Hui, C., \& McGeoch, M. A. (2007). Capturing the "droopytail” in the occupancy-abundance relationship. Ecoscience, 14, 103-108.

Hui, C., McGeoch, M. A., Reyers, B., le Roux, P. C., Greve, M., \& Chown, S. L. (2009). Extrapolating population size from the occupancy-abundance relationship and the scaling pattern of occupancy. Ecological Applications, 19, 2038-2048.

Hui, C., Terblanche, J. S., Chown, S. L., \& McGeoch, M. A. (2010). Parameter landscapes unveil the bias in allometric prediction. Methods in Ecology and Evolution, 1, 69-74.

Hui, C., Veldtman, R., \& McGeoch, M. A. (2010). Measures, perceptions and scaling patterns of aggregated species distributions. Ecography, 33, 95-102.

Johnson, C. N. (1998). Species extinction and the relationship between distribution and abundance. Nature, 394, 272-274.

Kendal, W. S. (2004). Taylor's ecological power law as a consquence of scale invariant exponential models. Ecological Complexity, 1, 193-209.

Lloyd, M. (1967). Mean crowding. Journal of Animal Ecology, 36, $1-30$.

Perry, J. N. (1981). Taylor's power law for dependence of variance on mean in animal populations. Applied Statistics, 30, 254-263.
Pielou, E. C. (1977). Mathematical ecology (2nd ed.). New York: Wiley \& Sons.

Plotkin, J. B., Chave, J. M., \& Ashton, P. S. (2002). Cluster analysis of spatial patterns in Malaysian tree species. American Naturalist, 160, 629-644.

Rodriguez, A., \& Delibes, M. (2002). Internal structure and patterns of contraction in the geographic range of the Iberian lynx. Ecography, 25, 314-328.

Roura-Pascual, N., Bas, J. M., \& Hui, C. (2010). The spread of the Argentine ant: Environmental determinants and impacts on native ant communities. Biological Invasions, 12, 2399-2412.

Shigesada, N., Kawasaki, K., \& Teramoto, E. (1979). Spatial segregation of interacting species. Journal of Theoretical Biology, 79, 83-99.

Skellam, J. G. (1951). Random dispersal in theoretical populations. Biometrika, 38, 196-218.

Taylor, L. R. (1961). Aggregation, variance and mean. Nature, 189, 732-735.

Wiens, J. A. (2000). Ecological heterogeneity: Ontogeny of concepts and approaches. In M. J. Hutchings, E. A. John, \& A. J. A. Stewart (Eds.), The ecological consequences of envrionmental heterogeneity (pp. 9-31). London: Blackwell.

Wiens, J. A. (2002). Riverine landscapes: Taking landscape ecology into the water. Freshwater Biology, 47, 501-515.

Wilson, R. J., Thomas, C. D., Fox, R., Roy, D. B., \& Kunin, W. E. (2004). Spatial patterns in species distributions reveal biodiversity change. Nature, 432, 393-396.

Wright, D. H. (1991). Correlations between incidence and abundance are expected by chance. Journal of Biogeography, 18, 463-466. 